\title{
METHODS FOR DIFFERENT ORDERS STRESSES ESTIMATION WITH DIFFRACTION METHODS
}

\author{
Elżbieta Gadalińska \\ Institute of Aviation, Materials and Structures Research Center, \\ al. Krakowska 110/114, 02-256 Warszawa, Poland
}

\begin{abstract}
The publication describes how diffraction methods and mathematical bases can be used for measurement of various types of stresses in single-phase and multiphase materials. Firstly, the paper defines the stresses and classifies them from the scale of their interactions point of view. Subsequently, the phenomenon of radiation diffraction on the crystalline lattice is presented including formulas describing this phenomenon and the dependencies enabling stress measurements. The key part of the paper is the description of one of the second order stress estimation methods based on diffraction data and a selfconsistent model.
\end{abstract}

Keywords: stress, diffraction methods for stress estimation, orders of stresses.

\section{INTRODUCTION: STRESS - DEFINITION AND CLASSIFICATION}

Stress can be defined as the measure of force density applied to the surface of an object. The stress state can be described by a tensor of rank two. In this analysis, it is assumed that the stress state in the specimen was generated by a set of forces as in Fig. 1. The forces acting on the specimen are distributed perpendicular to the surface of the specimen and in two shear directions. Each stress component $\sigma_{i j}$ can be described as a partial derivative of the force component and within the area of surface $j$ :

$$
\sigma_{i j}=\frac{\partial F_{i}}{\partial S_{j}}
$$

Let $\sigma_{11}, \sigma_{22}, \sigma_{33}$, represent the normal stress components, while $\sigma_{12}, \sigma_{13}, \sigma_{31}$, $\sigma_{23}, \sigma_{32}$ represent the shear components. The condition of the static balance state, which in this case corresponds to the equilibrium of moments, reduces the number of the components from 9 to 6 because $\sigma_{i j}=\sigma_{j i}$. 


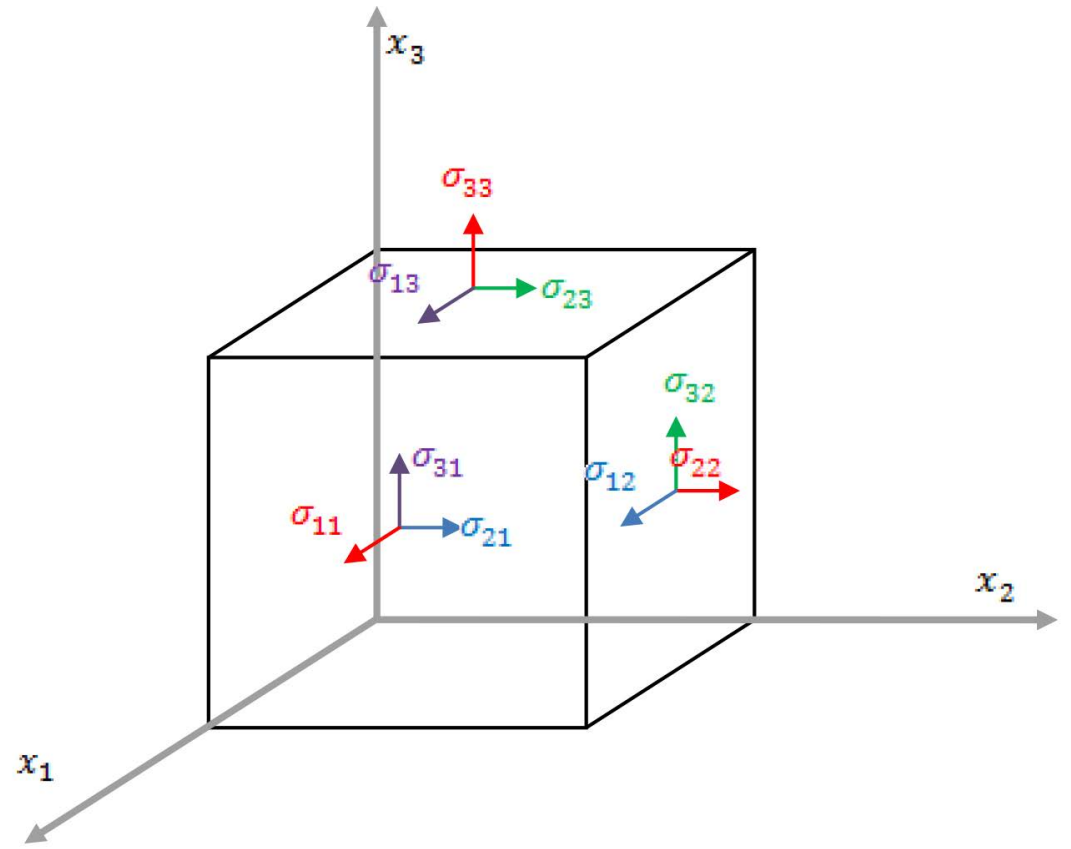

Fig. 1 The stress state in the infinitesimal cubic volume [1].

Of special interest are the residual stresses remaining in the material after specimen revealing. The most common reason for the increase in residual stresses is the heterogeneous plastic deformation, which can be single-axis or multi-axis and can be caused by machining, e.g. rolling, grinding or polishing. Residual stresses can also occur during heat treatment due to the temperature gradient or thermal the mismatch of the grains of the different phases that compose the material. Other causes may include phase transitions during heat treatment and hardening processes.

Residual stresses are very important due to the fact that their values add up together with the values of stresses induced by external forces applied to the object. Therefore, residual stress may contribute to damage acceleration in the material or a component. On the other hand, compressive residual stresses are generally desirable in the surface layer of most materials as they can extend the service life of a structure by reducing the impact of stresses applied. In order to introduce compressive stresses into the surface layer, various methods of material processing have been developed.

When analysing diffraction data, it is necessary to define the scale of the considerations. The reason for this is that lattice deformations at different levels have different effects on the diffraction image. Stresses can be classified into three 
types/orders depending on the scale of their impact on a structure. The first order stresses called macrostress are the mean stresses in the whole volume containing a large number of grains [2]. Stresses of this type are mainly induced by the heterogeneity of the process on a large scale, e.g. temperature gradient during machining. The mathematical definition of the first order stress is as follows:

where:

$$
\sigma_{i j}^{M}=\frac{1}{V_{A}} \int_{V_{A}} \sigma_{i j}(\boldsymbol{r}) d V=\sum_{g}^{N_{A}} f^{g} \sigma_{i j}^{g}
$$

$V_{A}$ - examined volume of the specimen,

$\sigma_{i j}(\boldsymbol{r})$ - local stress in position,

$f^{g}=\frac{V_{g}}{V_{A}}-g$ grains volume fraction,

$N_{A}-{ }$ total number of grains in $V_{A}$ volume,

$\sigma_{i j}^{g}$ - average stress value for $g$ grains within $V_{A}$ volume.

First order stresses can also be defined as phase stresses for the polycrystalline material, also called pseudo-stresses (weighed on all grains $g$ of the given $p h$ phase within the tested volume $V_{A}$ ), induced by various thermal and elastoplastic properties of phases or generated during the phase transition process:

$$
\sigma_{i j}^{p h}=\sum^{N_{p h}} f^{g} \sigma_{i j}^{g}
$$

where:

$N_{p h}$ - total numer of $p h$ phase grains.

The stress on a phase can be derived from the elastic phase response when the macrostress is applied to the sample $\left(\sigma_{i j}^{p h(e r)}\right)$ and/or from different thermal or plastic behaviour of the phases during sample processing leading to phase mismatch stresses $\left(\sigma_{i j}^{g(i c)}\right)$ [2], i.e.:

$$
\sigma_{i j}^{p h}=\sigma_{i j}^{p h(e r)}+\sigma_{i j}^{p h(i c)}
$$

The mismatch stresses remain in the sample even after the external forces are released.

In addition, the relationship between macrostress and stress in phase can be written as:

where:

$$
\sigma_{i j}^{M}=\sum_{p h}^{M} f^{p h} \sigma_{i j}^{p h}
$$


$f^{p h}=\frac{V_{p h}}{V_{A}}-$ volume fraction of examined phase $p h$, $\sigma_{i j}^{p h}$ - average stresses for phase $p h$,

$M$ - number of phases.

Second order stresses are the "deviation" from the first order stresses on the grains $\left(\sigma_{i j}^{g}\right)$. They are generated by the different orientation of the crystalline lattice with respect to the geometry of the sample, which in turn causes different behaviour of crystallites [2]. When external forces are applied to the material, the grain elastic response $\left(\sigma_{i j}^{g(e r)}\right)$ will depend on the grain orientation because the elastic constants of a single crystallite are anisotropic. Another reason for occurring of second order stresses is mechanical machining. During the plastic deformation of the polycrystalline material, differences in plastic deformation of differently oriented grains result in mismatch at the boundary leading to the formation of mismatch stress $\left(\sigma_{i j}^{g(i c)}\right)$. Second order stress may also occur during cooling/heating processes due to the anisotropy of the crystallite thermal expansion coefficients leading to grain volume decrease or increase in different directions in relation to the specimen geometry. The mathematical formula defining the second order stress is as follows:

$$
\sigma_{i j}^{I I g}=\sigma_{i j}^{g}-\sigma_{i j}^{I}
$$

where $\sigma_{i j}^{I}=\sigma_{i j}^{M}$ for one-phase materials and $\sigma_{i j}^{I}=\sigma_{i j}^{p h}$ for multi-phase materials.

Stresses on the grain $\sigma_{i j}^{g}$ can be expressed as the combination of stresses being the elastic response of the grain $\left(\sigma_{i j}^{g(e r)}\right)$ and those resulting from the mismatch of shape or volume of the individual grains to its surroundings $\left(\sigma_{i j}^{g(i c)}\right)[2]$ :

$$
\sigma_{i j}^{g}=\sigma_{i j}^{g(e r)}+\sigma_{i j}^{g(i c)}
$$

The stresses that define the material on the smallest scale are the stresses of the third order. They describe the level of homogeneity on the grain scale. Their source is local stress fields around defects in the crystalline lattice and can be defined as follows:

$$
\sigma_{i j}^{I I I}(\boldsymbol{r})=\sigma_{i j}(\boldsymbol{r})-\sigma_{i j}^{g}
$$


Changes in the diffraction image are different for different orders of stress. Stresses of first and second order cause the diffraction peak to shift in relation to the position of the peaks from stress free material. The second and third order stresses cause the diffraction peak to widen. For the summary of stress information for all orders see Table 1 [2]:

Table 1. Types of stresses and their origins [2].

\begin{tabular}{|c|c|c|c|c|c|}
\hline & \multicolumn{4}{|c|}{ SCALE } \\
\hline & & \multicolumn{2}{|c|}{ I order } & II order & III order \\
\hline & & single phase & multiphase & \multicolumn{2}{|c|}{ single and multiphase } \\
\hline \multirow{7}{*}{$\begin{array}{l}\text { Zu } \\
\text { 잉 }\end{array}$} & \multirow{4}{*}{$\begin{array}{l}\text { loads } \\
\text { long scale forces }\end{array}$} & \multicolumn{2}{|c|}{ macrostress } & \multirow{2}{*}{$x$} & \multirow{2}{*}{$x$} \\
\hline & & \multicolumn{2}{|c|}{$\sigma^{M}$} & & \\
\hline & & \multicolumn{3}{|c|}{ elastic stress } & \multirow{2}{*}{$x$} \\
\hline & & $x$ & $\sigma^{p h(e r)}$ & $\sigma^{I I g(e r}$ & \\
\hline & \multirow{2}{*}{$\begin{array}{l}\text { thermal processes } \\
\text { plastic processes }\end{array}$} & \multicolumn{3}{|c|}{ incompatibility stress } & \multirow{2}{*}{$x$} \\
\hline & & $x$ & $\sigma^{p h(i c}$ & $\sigma^{I I g(i c)}$ & \\
\hline & lattice imperfections & $x$ & $x$ & $x$ & $\sigma^{I I I}(r)$ \\
\hline
\end{tabular}

Fig. 2 illustrates the idea of stress classification in two-phase materials. The first order stresses (green line) are the mean macro scale stresses on the specimen. The first order stresses for the different phases of the material (black line for the first phase and purple one for the second) are directed in opposite directions in relation to the value of the mean macrostress. The stresses of the second order (orange line) are the deviation of a single grain for a given phase from the stress values of the first order. The stresses of the third order (red line) deviate from the mean value of grain stress. 


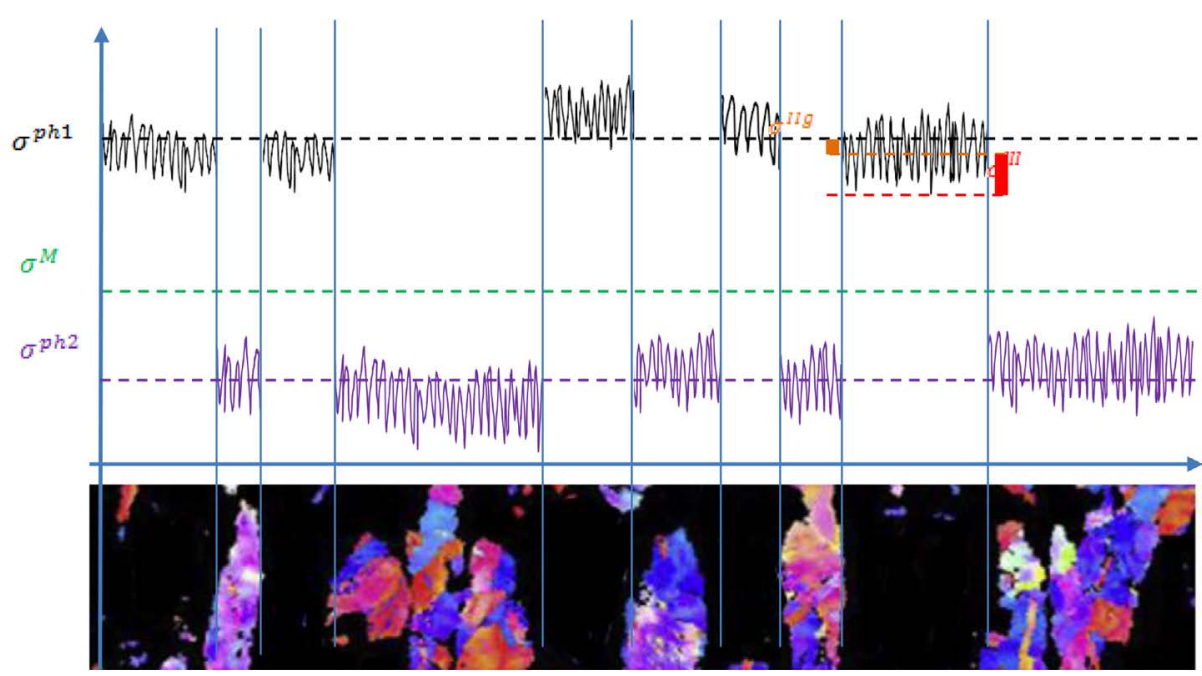

Fig. 2 Types of stresses in two phases material: I order stress for the whole specimen $\left(\sigma^{M}\right)$, I order stresses for different phases ( $\sigma^{p h 1}$ and $\left.\sigma^{p h 2}\right)$, II order stresses ( $\left.\sigma^{I I g}\right)$ as a deviation from I order stresses for phases and III order stress ( $\left.\sigma^{I I I}\right)$ as a deviation from the second order stress.

\section{THEORETICAL BACKGROUND OF DIFFRACTION PHENOMENON}

Until 1912, when Max von Laue conducted his experiment with the use of copper sulphate crystals, crystalline materials had only been suspected of having a periodical internal structure, which could be observed during the diffraction experiment (as in the case with other periodical structures, e.g. diffraction grating). The Laue experiment served as the stimulus for W. H. Bragga and his son W. L. Bragga to further explore this phenomenon. The phenomenon of diffraction is related to phase relationships occurring between two or more waves (electromagnetic but also other types, e.g. matter waves). When waves are diffracted on a periodical structure, the amplitude of the diffracted waves may be increased or decreased in relation to the incident wave depending on the phase relationship between the diffracted waves. The obvious conclusion following from the above is that the difference between the distances the diffracted beams have to pass is the key occurrence of the constructive (higher output wave amplitude) or destructive (lower amplitude) interference. The phenomenon of constructive interference occurring for non-periodic quasicrystalline structures is now also known. 


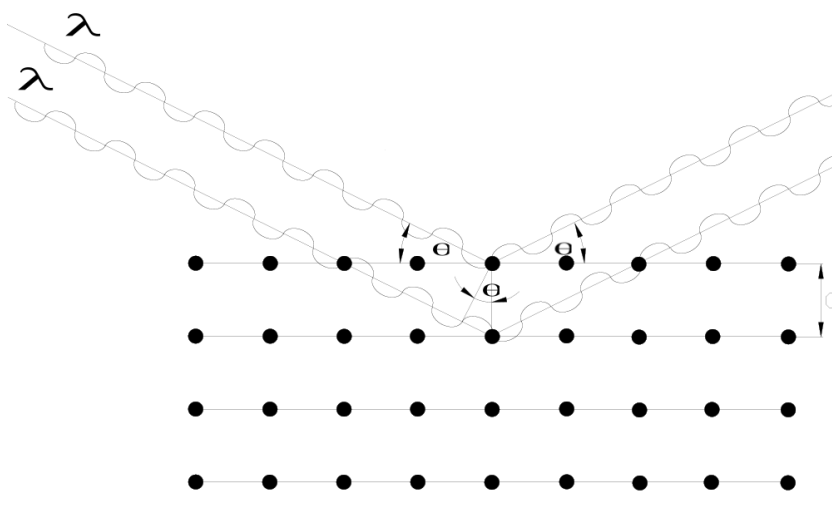

Fig. 3 The idea of diffraction on lattice planes.

Analysing Fig. 3, where diffraction is considered on the periodic crystalline lattice, it can be observed that constructive interference occurs when the difference in pathways between the incident and diffraction beams is equal to the total multiple $(n)$ wavelength $\lambda$. These considerations led to the formulation of Bragg's law:

$$
n \lambda=2 d \sin \theta
$$

Where $\theta$ is the angle between the incident / diffracted beam and the crystallographic plane while the angle of $2 \theta$ is the angle between the incident and diffracted beam.

A condition equivalent to Bragg's law may be expressed by the use of wave vectors $k_{l}$ i $k_{d}$ for incident and diffracted waves (Fig. 4).

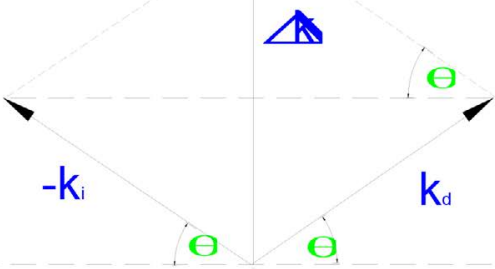

$\mathrm{K}_{\mathrm{i}}$

Fig. 4 Directions and angular dependencies of wave vectors associated with incident and diffracted beams. The wave vector as a sum of incident and diffracted ones is denoted by $\Delta k$. 
As can be seen in Fig. 4, the diffraction scattering vector is as follows:

$$
|\Delta \vec{k}|=\left|\vec{k}_{d}-\vec{k}_{i}\right|=\frac{4 \pi \sin \Theta}{\lambda}
$$

When Bragg's law is taken into account, the above can be written as:

$$
|\Delta \vec{k}|=\frac{2 \pi}{d_{h k l}}
$$

Then the distance between the plane $d_{h k l}$ will be linked to the vector of the reciprocal lattice vector $\vec{G}_{h k l}$ by the equation:

$$
\left|\vec{G}_{h k l}\right|=\frac{2 \pi}{d_{h k l}} .
$$

Taking these two equations into account, it can be concluded that constructive interference occurs when:

$$
|\Delta \vec{k}|=\left|\vec{G}_{h k l}\right|
$$

Since both $\Delta \vec{k}$ and $\vec{G}_{h k l}$ vectors are parallel to the crystallographic $h k l$ plane, a more general conclusion can be written as:

$$
\Delta \vec{k}=\vec{G}_{h k l}
$$

\section{DIFFRACTION METHODS USED FOR STRESS MEASUREMENTS}

\subsection{Isotropic specimen $-\sin ^{2} \psi$ method}

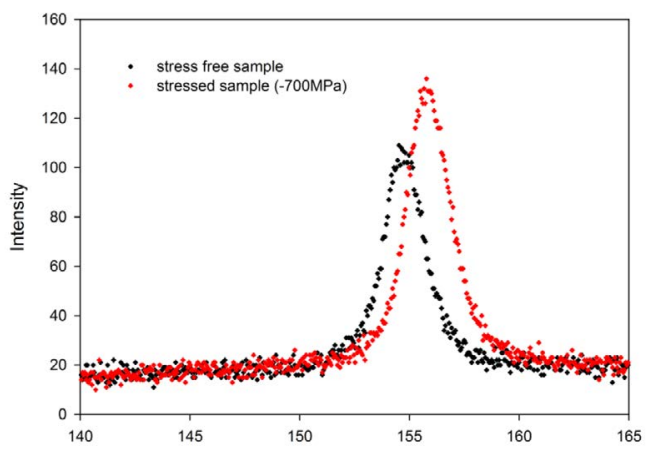

Fig. 5 The example of peak shift as a result of elastic strain of the lattice. Measurement performed on ferritic phase in 17-4PH stainless steel with chromium $X$-ray tube. 
The existence of stress in the material conjoins with a systematic increase or decrease in interplanar distances. Distances between the lattice planes can be measured by diffraction and Bragg's law. Based on Bragg's law, it can be deduced that if the distances between the $h k l$ planes increase, the $2 \theta$ angle decreases.

The most frequently diffraction stress measurements are carried out for one specific $h \mathrm{kl}$ plane. In this case, the sample rotates around the scattering vector $Q$ and for each sample orientation $\Omega-2 \Theta$ the position of the diffraction peak is determined. In order to describe the measurement, two reference systems should be defined: the first associated with the sample and the second, related to the measurement geometry, known as the laboratory system (Fig. 6).

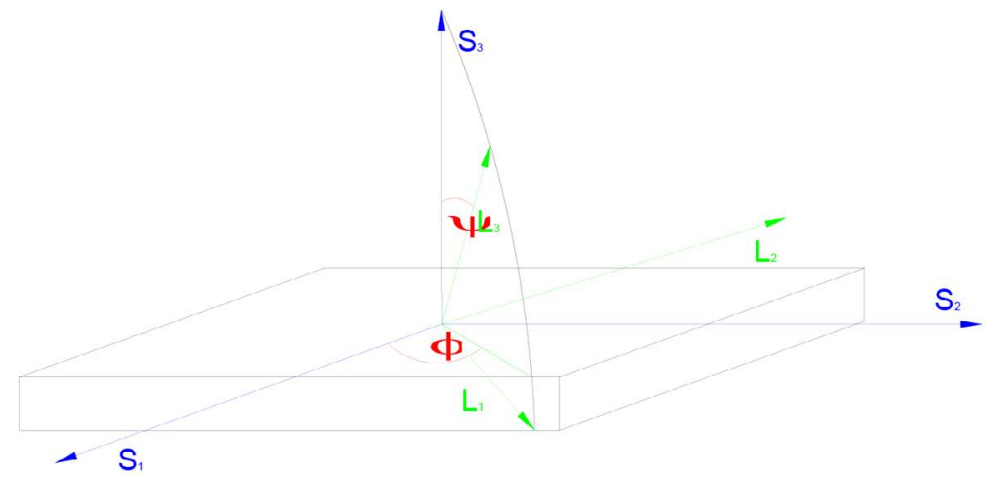

Fig. 6 Directions defined for the diffraction stress measurements. The specimen (S) and the laboratory $(L)$ systems are defined.

The idea behind the measurement is to tilt and rotate the sample in order to get information about the distance between the $h k l$ planes for different sets of angles $(\psi, \phi)$, i.e. $d_{h k l}(\psi, \phi)$ values for such $h k l$ planes for which the scattering vector is perpendicular. The average deformation value perpendicular to $L_{3}$ direction (for grains for which Bragg's law is fulfilled) is given by the equation:

$$
\left\langle\varepsilon_{33}(\psi, \phi)\right\rangle_{\{h k l\}}=\frac{\langle d(\psi, \phi)\rangle_{\{h k l\}}-d_{\{h k l\}}^{0}}{d_{\{h k l\}}^{0}}
$$

Using the generalised Hook's law, the relationship between the measured mean lattice strain values the $\left\langle\varepsilon_{33}(\psi, \phi)\right\rangle_{\{h k l\}}$ and the strain on the $\sigma_{i j}^{g \prime}$ grain is defined in the laboratory system $\boldsymbol{L}$ :

$$
\left\langle\varepsilon_{33}(\psi, \phi)\right\rangle_{\{h k l\}}=\left\langle s_{33 i j}^{g}{ }^{\prime} \sigma_{i j}^{g \prime}\right\rangle_{\{h k l\}}
$$


where $s_{33 i j}^{g}{ }^{\prime}$ is the appropriate component of the elastic compliance tensor for a single crystallite defined in the $\boldsymbol{L}$ laboratory system. The prim character was used to distinguish between the values defined in the $\boldsymbol{L}$ laboratory system (only in the notation of the mean deformation $\left\langle\varepsilon_{33}(\psi, \phi)\right\rangle_{\{h k l\}}$ measured along the scattering vector the prim character was omitted). The sign of average value $\langle\ldots\rangle$ is very important, as it determines the average value of the interplanar spacing for a special group of diffracting grains that comply with Bragg's law. Despite the fact that the averaging operation is not commutative with a multiplication and the stresses in eq. 16 are not averaged after all grains in the sample under consideration, the relationship between the measured lattice strain and the stress of the first order $\sigma_{i j}^{I \prime}$ in the examined phase can be described as:

$$
\left\langle\varepsilon_{33}(\psi, \phi)\right\rangle_{\{h k l\}}=R_{i j}(\psi, \phi) \sigma_{i j}^{I}
$$

where $R_{i j}(\psi, \phi)$ are diffraction elastic constants (DECs) and $\sigma_{i j}^{I}{ }^{\prime}$ are the first order stresses becoming from different sources, i.e. in the case of a single-phase material $\sigma_{i j}^{I{ }^{\prime}}=\sigma_{i j}^{M^{\prime}}$ and in the case of the multiphase material $\sigma_{i j}^{I}{ }^{\prime}=\sigma_{i j}^{p h^{\prime}}=\sigma_{i j}^{p h(e r) r}+\sigma_{i j}^{p h(i c){ }^{\prime}}$.

It should be noted that the DECs' values generally depend on angles $(\psi, \phi)$ and $\mathrm{hkl}$ Miller coefficients, while for the quasi-isotropic sample (without texture) there is no reason for angle dependence. Replacement $R_{i j}=\left\langle s_{33 i j}{ }^{\prime}\right\rangle_{\{h k l\}}$ is only justified if the stress values are identical for all crystallites in the sample (i.e. when the Reuss model is used). As the first and easiest case, a formula will be considered that links the state of stress in the specimen in a given direction with the lattice strain in the quasi-isotropic specimen without texture. A polycrystalline sample can be considered as isotropic only on a macro-scale because within a single grain its elastic anisotropy will always be observed. Due to the cylindrical symmetry of the set of diffracting grains, the compliance tensor can be defined in the form of Voigt notation [1]:

$$
S_{m n}{ }^{\prime}=\left[\begin{array}{cccccc}
S_{11}{ }^{\prime} & S_{12}{ }^{\prime} & S_{13}{ }^{\prime} & 0 & 0 & 0 \\
S_{12}{ }^{\prime} & S_{11}{ }^{\prime} & S_{13^{\prime}} & 0 & 0 & 0 \\
S_{13}{ }^{\prime} & S_{13}{ }^{\prime} & S_{33^{\prime}} & 0 & 0 & 0 \\
0 & 0 & 0 & S_{44}{ }^{\prime} & 0 & 0 \\
0 & 0 & 0 & 0 & S_{44}{ }^{\prime} & 0 \\
0 & 0 & 0 & 0 & 0 & S_{66}{ }^{\prime}
\end{array}\right]
$$


Using this matrix, the general equation bonding stress and strain may be written [1]:

$$
\left\langle\varepsilon_{33}(\psi, \phi)\right\rangle_{\{h k l\}}=S_{31}{ }^{\prime} \sigma_{11}^{I}{ }^{\prime}+S_{32}{ }^{\prime} \sigma_{22}^{I}{ }^{\prime}+S_{33}{ }^{\prime} \sigma_{33}^{I}{ }^{\prime}=S_{13}{ }^{\prime} \sigma_{11}^{I}{ }^{\prime}+S_{13}{ }^{\prime} \sigma_{22}^{I}{ }^{\prime}+S_{33}{ }^{\prime} \sigma_{33}^{I}{ }^{\prime}
$$

The three components of this equation were neglected because $S_{34}{ }^{\prime}=S_{35}{ }^{\prime}=S_{36}{ }^{\prime}=0$.

Assuming the two-dimensional state of stress on the surface of the specimen (as for X-ray measurements), i.e.: $\sigma_{11}^{I} \neq 0, \sigma_{22}^{I} \neq 0, \sigma_{33}^{I}=\sigma_{12}^{I}=\sigma_{13}^{I}=\sigma_{23}^{I}=0$; defined in relation to the sample reference system $S$ and using the direction cosine, the state of stress in the laboratory reference system may be described by means of three equations [1]:

$$
\begin{gathered}
\sigma_{11}^{I}{ }^{\prime}=\sigma_{11}^{I} \cos ^{2} \phi \cos ^{2} \psi+\sigma_{22}^{I} \sin ^{2} \phi \cos ^{2} \psi \\
\sigma_{22}^{I}{ }^{\prime}=\sigma_{11}^{I} \sin ^{2} \phi+\sigma_{22}^{I} \cos ^{2} \phi \\
\sigma_{33}^{I}{ }^{\prime}=\sigma_{11}^{I} \cos ^{2} \phi \sin ^{2} \psi+\sigma_{22}^{I} \sin ^{2} \phi \sin ^{2} \psi
\end{gathered}
$$

where the stress tensor $\sigma_{i j}^{I}$ (without prim character) is defined in relation to the sample reference system $\boldsymbol{S}$.

Applying the equations eq. 19, eq. 20, eq. 21 and eq. 22 it can be written:

$$
\begin{gathered}
\sigma_{33}^{I}{ }^{\prime}=\sigma_{11}^{I} \cos ^{2} \phi \sin ^{2} \psi+\sigma_{22}^{I} \sin ^{2} \phi \sin ^{2} \psi \\
+S_{13}{ }^{\prime}\left(\sigma_{11}^{I} \sin ^{2} \phi+\sigma_{22}^{I} \cos ^{2} \phi\right)+S_{33}{ }^{\prime}\left(\sigma_{11}^{I} \cos ^{2} \phi \sin ^{2} \psi+\sigma_{22}^{I} \sin ^{2} \phi \sin ^{2} \psi\right)
\end{gathered}
$$

Applying the trigonometric identity $\cos ^{2} \psi=1-\sin ^{2} \psi$ :

$$
\begin{aligned}
\left\langle\varepsilon_{33}(\psi, \phi)\right\rangle_{\{h k l\}} & =S_{13}{ }^{\prime}\left(\sigma_{11}^{I}+\sigma_{22}^{I}-\sigma_{11}^{I} \cos ^{2} \phi \sin ^{2} \psi-\sigma_{22}^{I} \sin ^{2} \phi \sin ^{2} \psi\right) \\
& +S_{33}{ }^{\prime}\left(\sigma_{11}^{I} \cos ^{2} \phi \sin ^{2} \psi+\sigma_{22}^{I} \sin ^{2} \phi \sin ^{2} \psi\right) \\
\left\langle\varepsilon_{33}(\psi, \phi)\right\rangle_{\{h k l\}}= & \left({S_{33}}^{\prime}-S_{13}{ }^{\prime}\right)\left(\sigma_{11}^{I} \cos ^{2} \phi+\sigma_{22}^{I} \sin ^{2} \phi\right) \sin ^{2} \psi+S_{13}\left(\sigma_{11}^{I}+\sigma_{22}^{I}\right)
\end{aligned}
$$

substituting:

$$
S_{33}{ }^{\prime}-S_{13}{ }^{\prime}=\frac{1}{2} s_{2} \text { i } S_{13}{ }^{\prime}=s_{1}
$$


the following equation is obtained:

$$
\left\langle\varepsilon_{33}(\psi, \phi)\right\rangle_{\{h k l\}}=\frac{1}{2} s_{2}\left(\sigma_{11}^{I} \cos ^{2} \phi+\sigma_{22}^{I} \sin ^{2} \phi\right) \sin ^{2} \psi+s_{1}\left(\sigma_{11}^{I}+\sigma_{22}^{I}\right)
$$

Using the formula for the interplanar distance in deformed crystal lattice (eq. $15)$ :

$$
\langle d(\psi, \phi)\rangle_{\{h k l\}}=\left[\frac{1}{2} s_{2}\left(\sigma_{11}^{I} \cos ^{2} \phi+\sigma_{22}^{I} \sin ^{2} \phi\right) \sin ^{2} \psi+s_{1}\left(\sigma_{11}^{I}+\sigma_{22}^{I}\right)\right] d_{\{h k l\}}^{0}+d_{\{h k l\}}^{0}
$$

Relations for $\left\langle\varepsilon_{33}(\psi, \phi)\right\rangle_{\{h k l\}}$ and $\langle d(\psi, \phi)\rangle_{\{h k l\}}$ are linear in relation to $\sin ^{2} \psi$. An example is shown in the figure below.

Fig. 7 Typical $\sin ^{2} \psi$ plot obtained for 17-4PH stainless steel specimen.

The slope of the straight line determined by this method shall be proportional to the stress measured in the $\phi$ direction. For cases where the values of $\sigma_{33}^{I}, \sigma_{13}^{I}$ and $\sigma_{23}^{I}$ are negligible (i.e. for neutron or synchrotron radiation when internal parts of the material are tested), the equation for $\left\langle\varepsilon_{33}(\psi, \phi)\right\rangle_{\{h k l\}}$ assumes the following formula:

$$
\begin{aligned}
\left\langle\varepsilon_{33}(\psi, \phi)\right\rangle_{\{h k l]} & =\frac{1}{2} s_{2}\left(\sigma_{11}^{I} \cos ^{2} \phi+\sigma_{12}^{I} \sin 2 \phi+\sigma_{22}^{I} \sin ^{2} \phi\right) \sin ^{2} \psi \\
& +\frac{1}{2} s_{2} \sigma_{33}^{I} \cos ^{2} \psi+s_{1}\left(\sigma_{11}^{I}+\sigma_{22}^{I}+\sigma_{33}^{I}\right)+\frac{1}{2} s_{2}\left(\sigma_{13}^{I} \cos \phi+\sigma_{23}^{I} \sin \phi\right) \sin 2 \psi
\end{aligned}
$$

and, consequently:

$$
\begin{aligned}
\langle d(\psi, \phi)\rangle_{\{h k l\}}= & {\left[\frac{1}{2} s_{2}\left(\sigma_{11}^{I} \cos ^{2} \phi+\sigma_{12}^{I} \sin 2 \phi+\sigma_{22}^{I} \sin ^{2} \phi\right) \sin ^{2} \psi\right.} \\
& \left.+\frac{1}{2} s_{2} \sigma_{33}^{I} \cos ^{2} \psi+s_{1}\left(\sigma_{11}^{I}+\sigma_{22}^{I}+\sigma_{33}^{I}\right)+\frac{1}{2} s_{2}\left(\sigma_{13}^{I} \cos \phi+\sigma_{23}^{I} \sin \phi\right) \sin 2 \psi\right] d_{\{h k l\}}^{0} \\
\langle d(\psi, \phi)\rangle_{\{h k l\}}= & {\left[\frac{1}{2} s_{2}\left\{\left(\sigma_{11}^{I}-\sigma_{33}^{I}\right) \cos ^{2} \phi+\sigma_{12}^{I} \sin 2 \phi+\left(\sigma_{22}^{I}-\sigma_{33}^{I}\right) \sin ^{2} \phi\right)\right\} \sin ^{2} \psi+s_{1}\left(\sigma_{11}^{I}+\sigma_{22}^{I}\right.} \\
& \left.\left.+\sigma_{33}^{I}\right)+\frac{1}{2} s_{2}\left(\sigma_{13}^{I} \cos \phi+\sigma_{23}^{I} \sin \phi\right) \sin 2 \psi\right] d_{\{h k l\}}^{0}+d_{\{h k l\}}^{0}
\end{aligned}
$$

In the most general case (anisotropic sample without special symmetry) the general equation eq. 17 should be used. In this case, the determination of Rij, which depends on the measuring direction, is essential.

\subsection{Multireflection method - anizotropic specimens with texture}

In a standard X-ray experiment, it is usually assumed that the stress component perpendicular to the specimen surface $\sigma_{33}^{I}$ is equal to 0 . The key feature of the $\sin ^{2} \psi$ method is measuring the mean interplanar spacing 
of $\langle d(\psi, \phi)\rangle_{\{h k l\}}$. These values are averaged only for grains participating in diffraction, i.e. for which the orientation of the scattering vector is perpendicular to the crystallographic planes $h k l$. Therefore, the analysis method with the use of quasi-isotropic elasticity constants $s_{1}$ and $s_{2}$ is adequate when the second order stresses and texture in the test sample are negligible. In this case, and when the stress state is of the two-axial nature (i. e. when $\sigma_{13}^{I}=\sigma_{23}^{I}=0$ ) eq. 29 can be illustrated as a linear dependence (Fig. 7). If the shear stresses $\sigma_{13}^{I}$ and $\sigma_{23}^{I}$ are significant in the specimen, the splitting phenomenon occurs (which consists in curvature of the plots $\sin ^{2} \psi$ dla $\varphi$ and $\varphi+180^{\circ}$ in opposite directions) [2]. Moreover, in the standard version of this method, the observed diffraction peaks correspond only to single $h k l$ planes and the measured lattice strains do not represent the whole volume of the tested sample [2].

A more general case occurs when a zero stress value of $\sigma_{33}^{I}$ cannot be assumed, i.e. when neutron or synchrotron radiation that penetrates deep into the material is used. Then the differences $\left(\sigma_{11}^{I}-\sigma_{33}^{I}\right)$ and $\left(\sigma_{11}^{I}-\sigma_{22}^{I}\right)$ are determined on the basis of the standard $\sin ^{2} \psi$ (cf. eq. 29). In addition, in the case of the significant texture, anisotropic elastic constants $\left(s_{1}\right.$ and $\left.s_{2}\right)$ cannot be used because DECs depend on the orientation distribution function and change with $\phi$ i $\psi$ angles. The result of the above is that $\langle d(\psi, \phi)\rangle_{\{h k l\}}$ function with respect to $\sin ^{2} \psi$ changes its form - it is no longer a linear or an elliptical function, but a complex nonlinearity is observed, i.e. a more general equation is necessary to describe changes in the interplanar distance [2]:

$$
\langle d(\psi, \phi)\rangle_{\{h k l\}}=\left[F_{i j}(h k l, \phi, \psi) \sigma_{i j}^{I}\right] d_{\{h k l\}}^{0}+d_{\{h k l\}}^{0}
$$

where:

$$
\langle d(\psi, \phi)\rangle_{\{h k l\}}=\left[F_{i j}(h k l, \phi, \psi) \sigma_{i j}^{I}\right] d_{\{h k l\}}^{0}+d_{\{h k l\}}^{0}
$$

$F_{i j}^{I}(h k l, \phi, \psi)$ - diffraction elastic constants for anisotropic material or for a given phase of the multiphase material, usually called X-ray stress factors $\mathrm{XSF} ; \gamma_{i j}$ results from the transformation of the sample reference system into the laboratory reference system:

$$
\gamma_{i j}=\left[\begin{array}{ccc}
\cos \phi \cos \psi & \sin \phi \cos \psi & -\sin \psi \\
-\sin \phi & \cos \phi & 0 \\
\cos \phi \sin \psi & \sin \phi \sin \psi & \cos \psi
\end{array}\right]
$$


Different $h \mathrm{kl}$ reflections can be used simultaneously in the multireflection method, the idea of which is to obtain $\sigma_{i j}^{I}$ and $a_{0}$ (when the regular structure is tested) based on the calculated diffraction elastic constants $F_{i j}(h k l, \phi, \psi)$ and after the transformation of Eq. 30 by substitution:

$$
\begin{gathered}
\langle a(\phi, \psi)\rangle_{\{h k l\}}=\langle d(\psi, \phi)\rangle_{\{h k l\}} \cdot \sqrt{h^{2}+k^{2}+l^{2}} \\
a^{0}=d_{\{h k l\}}^{0} \cdot \sqrt{h^{2}+k^{2}+l^{2}}
\end{gathered}
$$

The resulting equation is:

$$
\langle a(\phi, \psi)\rangle_{\{h k l\}}=\left[F_{i j}(h k l, \phi, \psi) \sigma_{i j}^{I}\right] a^{0}+a^{0}
$$

As a result of this transformation, instead of many values of unstressed interplanar spacings $d_{\{h k l\}}^{0}$, only one parameter of the unstressed crystalline lattice (i.e. $a^{0}$ ) is used in the analysis [2].

The advantage of the multireflection method is that the results obtained are representative for a large number of grains, to a much greater extent than it is in the case of the single reflection method.

\subsection{Diffraction methods used for estimation of second order stresses}

The effect of the second order stresses on the $\sin ^{2} \psi$ plots consists in the introduction of non-linearity into these functions. A number of methods have been developed to calculate approximate values of second order stress. One of them is the method proposed and developed by Baczmański [3] - [4]. In this method, the quantitative approximation of the first and second order stresses is the result of reference to the diffraction data and to the self-consistent model. In addition, the multireflection method enables obtaining more representative data by the simultaneous fitting of different $h \mathrm{kl}$ reflections.

The equation that conjoins the strains and stresses, including the first type of stresses (macrostresses or phase stresses) and mismatch stresses, is [2]:

$$
\langle\varepsilon(\phi, \psi)\rangle_{\{h k l\}}=F_{m n}(h k l, \phi, \psi) \sigma_{m n}^{I}+\left\langle\gamma_{3 m} \gamma_{3 n} s_{m n i j} \sigma_{i j}^{I I g(i c)}\right\rangle_{\{h k l\}}
$$

It should be noted here that the second order mismatch stresses $\sigma_{i j}^{I I g(i c)}$ do not disappear after the specimen is unloaded. In addition, their value can be approximated by model-based calculations of the self-consistent model, in 
which the input data include the type of reinforcement mechanism that occurs during plastic deformation. Since the description of the hardening process is complicated (requires consideration of additional empirical laws) and not always possible to simulate with the use of the model, it is usually assumed that values $\left(\overline{\left.\sigma_{\imath \jmath}^{I I g(l c)}(\mathbf{g})\right)}\right.$ approximated by the model values, which are not equal to the actual stresses of the second order but proportional to them, i.e.:

$$
\sigma_{i j}^{I I g(i c)}(\mathbf{g})=q \overline{\sigma_{l \jmath}^{I I g(l c)}(\mathbf{g})}
$$

These two values, i.e. actual and model second order stress values, are related to each other by the scale parameter $q$, which is not dependent on the grain orientation $\mathbf{g}$.

Taking into account Eq. 37, Eq. 30 and Eq. 35 will change:

$$
\begin{gathered}
\langle d(\psi, \phi)\rangle_{\{h k l\}}=\left[F_{i j}(h k l, \phi, \psi) \sigma_{i j}^{I}+q \overline{\left\langle\gamma_{3 m} \gamma_{3 n} s_{m n l \jmath} \sigma_{l j}^{I I g(l c)}(\mathbf{g})\right\rangle_{\{h k l\}}}\right] d_{\{h k l\}}^{0}+d_{\{h k l\}}^{0} \\
\langle a(\phi, \psi)\rangle_{\{h k l\}}=\left[F_{i j}(h k l, \phi, \psi) \sigma_{i j}^{I}+q \overline{\left\langle\gamma_{3 m} \gamma_{3 n} s_{m n l} \sigma_{l \jmath}^{I I g(l c)}(\mathbf{g})\right\rangle_{\{h k l\}}}\right] a^{0}+a^{0}
\end{gathered}
$$

The alignment procedure using the least square method provides information on the parameters, i.e. the first order stress $\sigma_{i j}^{I}$, the scale parameter $q$, the lattice parameter $a^{0}$ and the interplanar spacing $d_{\{h k l\}}^{0}\left(\right.$ lub $\left.a_{\{h k l\}}^{0}\right)$ for the unstressed specimen. If the value of $q$ parameter is close to 1 , model predictions of second order mismatch stresses are correct while in the case when $q<1$, model values will be overestimated, which is associated with stress relaxation in the actual sample. When $q \neq 1$, the reinforcement process was not correctly predicted. If the value $q$ is determined by the procedure of the least square procedure, the actual value of second order stresses may be determined by multiplying the model values, cf. Eq. 37 


\section{REFERENCES}

[1] K. Wierzbanowski, Rzeczywista struktura materiałów, provisory notes for AGH students, Kraków.

[2] A. Baczmański, Stress fields in polycrystalline materials studied using diffraction and self-consistent modeling. Kraków: Wydział Fizyki i Informatyki Stosowanej AGH, 2005.

[3] A. Baczmański, K. Wierzbanowski, W. G. Haije, R. B. Helmholdt, G. Ekambaranathan, and B. Pathiraj, Diffraction Elastic Constants for Textured Materials - Different Methods of Calculation, Cryst. Res. Technol., vol. 28, no. 2, pp. 229-243, Jan. 1993.

[4] A. Baczmanski, K. Wierzbanowski, and P. Lipiński, Determination of Residual Stresses in Plastically Deformed Polycrystalline Material, Mater. Sci. Forum, vol. 157-162, pp. 2051-2058, 1994. 